\title{
A SPECTROPHOTOMETRIC METHOD FOR THE RAPID ESTIMATION OF BLOOD OXYGEN SATURATION, CONTENT, AND CAPACITY
}

BY

\author{
I. C. RODDIE,* J. T. SHEPHERD, AND R. F. WHELAN \\ From the Department of Physiology, the Queen's L'niversity of Belfast
}

(RECEIVED FOR PUBLICATION OCTOBER 20, 1956)

Various photometric and spectrophotometric methods have been devised for the estimation of blood oxygen saturation (Nicolai, 1932; Kramer, 1934; Matthes and Gross, 1939; Wood, 1950; Handforth, 1952; Wade, Bishop, Cumming, and Donald, 1953; Molyneux and Pask, 1955), and the principles and basic requirements have been clearly defined (Drabkin, 1950; Wood, 1950; Kramer, Elam, Saxton, and Elam, 1951). In some methods whole blood is used, while in others the blood is first haemolysed. The use of cuvette oximeters for whole blood, such as those described by Drabkin and Austin (1935), Wood (1950), and Kramer et al. (1951), enables rapid serial estimations or continuous recording of blood oxygen saturation to be made, while mo:t of the methods utilizing haemolysed blood are relatively time-consuming and usually require the removal, filling, and replacement of the cuvette in the instrument (Hickam and Frayser, 1949; Nahas, 1951; Wade et al., 1953; Molyneux and Pask, 1955). However, the use of haemolysed rather than whole blood in such techniques has the advantage that it is a fluid of simpler optical properties. In whole blood the intact red cells cause reflection and scattering of the incident light, and intracellular haemoglobin causes magnified light absorption (Kramer et al., 1951).

This paper describes a simple and rapid method, employing haemolysed blood, for the estimation not only of blood oxygen saturation but also of oxygen content and capacity. The use of a cuvette of small capacity and good wash-out characteristics enables blood samples to be passed in quick succession through the cuvette without removing it from the instrument. A standard spectrophotometer is employed and requires no modification other than the fitting of the cuvette into the holder supplied.

\footnotetext{
- Walter Dixon Memorial Scholar (British Medical Association).
}

\section{The Cuvette}

The exact shape and dimensions of the cuvette are not critical, since the instrument is calibrated and used with the cuvette mounted in a fixed position in the cell holder. A shape of cuvette which is satisfactory when used with the "unicam" S.P. 400 or S.P. 350 absorptiometer (Unicam Instruments Ltd., Arbury Works, Cambridge, England) is a flat chamber of "pyrex" glass blown to shape between asbestos sheets so that it has a chamber depth of about $1 \mathrm{~mm}$. (Fig. 1). The inlet and outlet tubes are about $2 \mathrm{~mm}$. in internal diameter. The cuvette is mounted with dental wax in the ebonite holder for rectangular cells supplied with the instrument. Rubber or polythene tubing is attached to the glass tubes leading to and from the chamber and passes through a hole in the top of the holder which is then sealed with plasticine or dental wax. The tubes and that part of the chamber not in the light path are coated with matt black paint. By means of the tubing the chamber can be filled and flushed without removal from the instrument. The optical properties are thus constant and it is not necessary that the walls be absolutely flat. About $0.6 \mathrm{ml}$. of solution is sufficient to fill the cuvette and inlet tubing; and $2 \mathrm{ml}$. is sufficient to flush it out. The chamber is filled via the tube which enters at the bottom in order to expel air bubbles.

\section{Details of the Technique}

Percentage Oxygen Saturation.-The percentage oxygen saturation of a given sample of blood is derived from the ratio $D_{R}-D_{T} / D_{R}-D_{0}$, where $D_{R}, D_{0}$, and $D_{T}$ represent the optical densities of the fully reduced, the fully oxygenated, and the test samples respectively, determined at a wavelength of $660 \mathrm{~m} \mu$. The value so obtained is converted into percentage saturation using the calibration curve shown in Fig. 2 .

The instrument is set to read zero optical density with the cuvette filled with saline. One millilitre of the blood under examination (test sample) is drawn into a $3 \mathrm{ml}$. nylon syringe and is followed by $1 \mathrm{ml}$. of a $2 \%$ $(w / v)$ solution of white saponin (B.D.H.) in $0.1 \%$ sodium carbonate. A little mercury is then drawn into the syringe for mixing and, after shaking for five to 10 sec., the solution is injected into the cuvette. A 


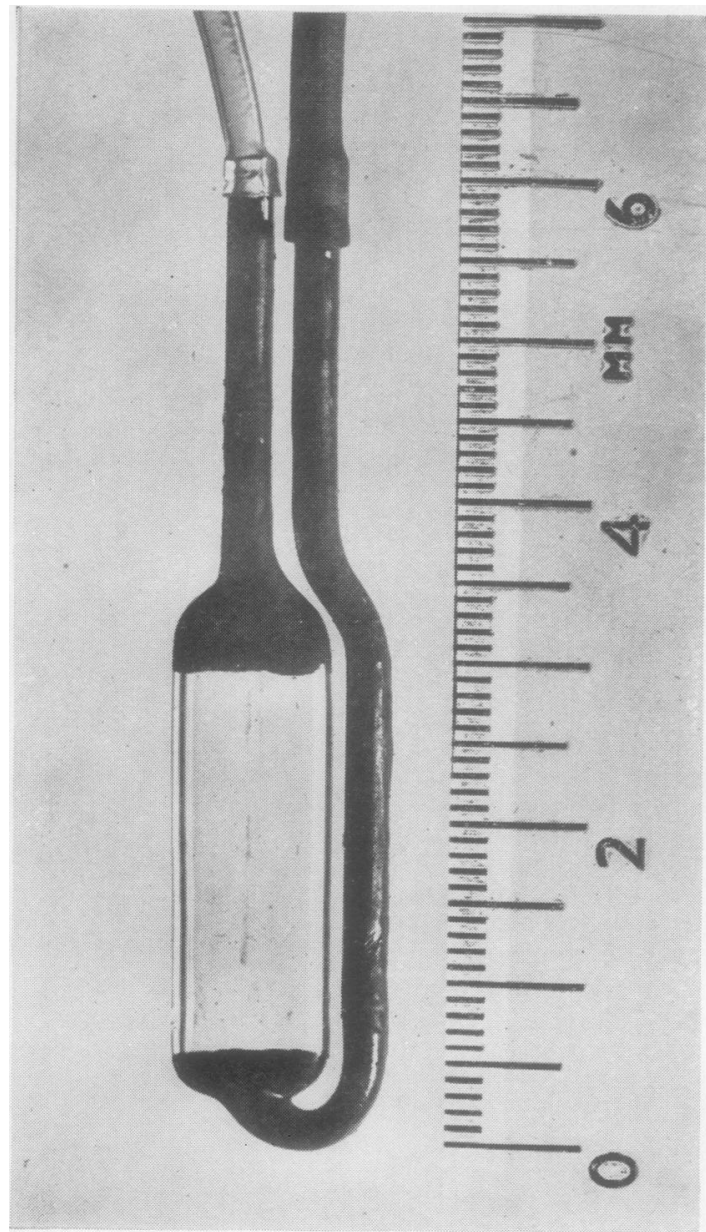

Fig. 1.-The cuvette.

sample of fully oxygenated blood is then drawn into a syringe and the same technique applied. To determine the reading for fully reduced blood the same dilution (1:1) of a blood sample is used, but hydrosulphite is added to the diluting solution. This is made by adding under paraffin $1 \mathrm{ml}$. of freshly prepared saturated sodium hydrosulphite (prepared under paraffin) to $6.5 \mathrm{ml}$. of sodium carbonate-saponin solution. The cuvette is flushed out with 5 to $10 \mathrm{ml}$. of saline between each observation.

Oxygen Capacity.-The oxygen capacity of blood is derived from the difference in optical density between the fully reduced and fully oxygenated samples at the same wavelength of $660 \mathrm{~m} \mu$, this value being converted to oxygen capacity in vol. \% by the use of a calibration curve (Fig. 3). A capacity calibration curve must be constructed for each cuvette, since $D_{R}-D_{0}$ for a given haemoglobin value will depend on the thickness of the cuvette.

Oxygen Content.-The oxygen content is derived from the oxygen saturation and capacity.

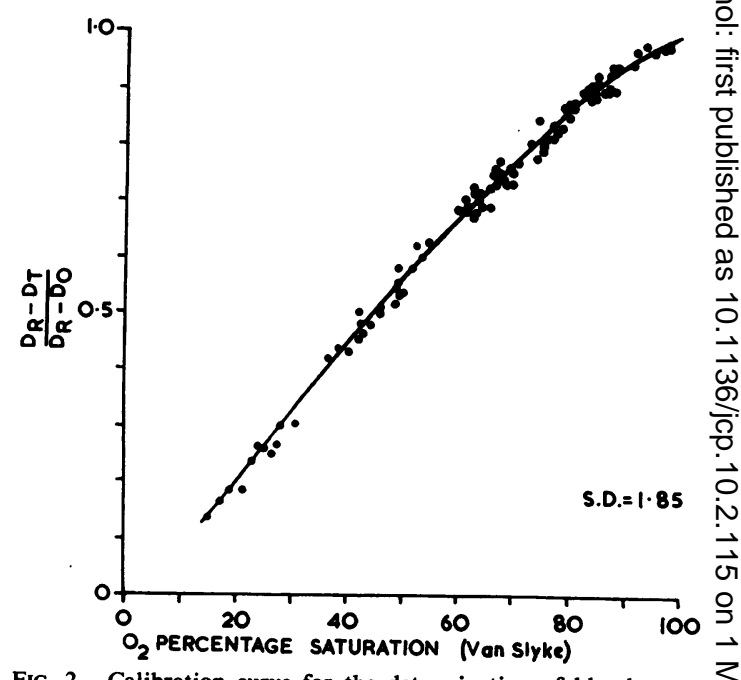

FIG. 2.-Calibration curve for the determination of blood oxygen saturation. The curve is based on 100 estimations of oxyger $K$ saturation by the photoelectric and Van Slyke methods. $\mathbf{D}_{\mathrm{R}}=\overrightarrow{\mathrm{C}}$ optical density of the reduced sample. $D_{T}=$ optical density of the test sample. $D_{0}=$ optical density of the oxygenated sample. S.D. = standard deviation of the individual points from the curve.

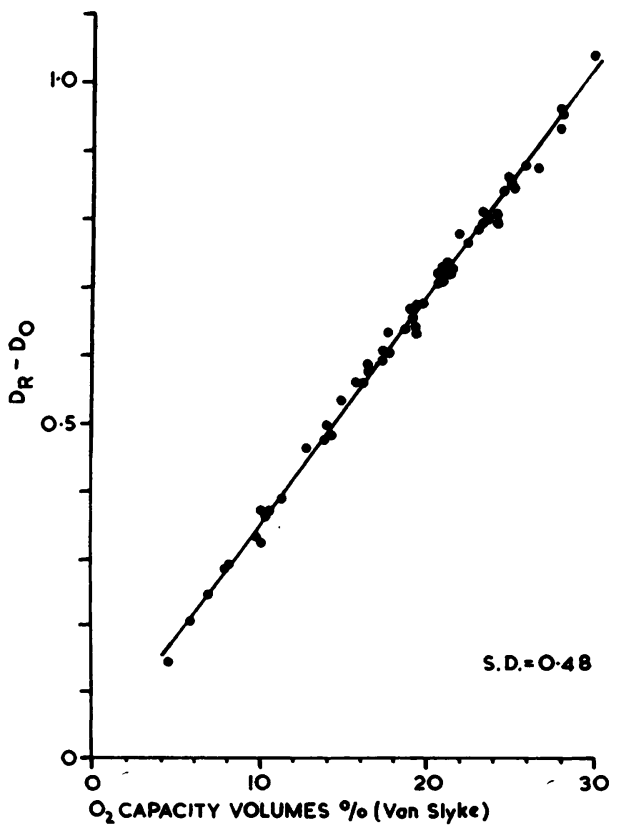

FIG. 3.-Calibration curve for the determination of blood oxygen $\mathbb{D}$ capacity from photoelectric optical density measurements. The curve is based on 57 estimations of oxygen capacity by the photoelectric and Van Slyke methods. $D_{R}=$ optical density of the reduced sample. $D_{0}=$ optical density of the oxygenated sample. The regression line through these points is shown.? S.D. = standard deviation from the regression line. 


\section{Notes on the Method}

(a) The dilution of the blood samples with equal volumes of carbonate saponin solution does not result in gas exchange of any consequence between the blood and the diluting fluid. The amount of saponin used was found to haemolyse $1 \mathrm{ml}$. blood in $5 \mathrm{sec}$. In preliminary experiments the synthetic detergent "lissapol" was used to produce haemolysis of the blood samples (Wade et al., 1953; Molyneux and Pask, 1955), but was discontinued as it produced opacity with hydrosulphite.

(b) Using 1:1 dilution of the blood samples, the chamber depth and wavelength were selected so as to give a large difference in the deflection of the galvanometer between oxygenated and reduced blood. With a chamber depth of about $1.0 \mathrm{~mm}$., a wavelength of $660 \mathrm{~m} \mu$, and using the S.P. 400 absorptiometer, the transmission compared with saline ranged from $70 \%$ to $76 \%$ for fully oxygenated normal blood, and from $12 \%$ to $16 \%$ for reduced blood. The cuvette dimensions are not critical and replacing the cuvette by another of somewhat different dimensions does not affect the calibration for percentage oxygen saturation, although it would necessitate recalibration for capacity.

(c) The greatest difference between the percentage transmission of oxygenated and reduced haemoglobin is found in the red range (Horecker, 1943). In the present method a wavelength setting of $660 \mathrm{~m} \mu$ was used in preference to the more usual one of $630 \mathrm{~m} \mu$, since, although the difference in optical density between reduced and oxygenated blood is less at $660 \mathrm{~m} \mu$, the percentage light transmission, and hence the galvanometer sweep, was greater.

(d) The amount of sodium hydrosulphite necessary to reduce the haemoglobin in $1 \mathrm{ml}$. of blood is fairly critical. Too much causes turbidity and too little causes incomplete reduction. The minimum amount was found to be that contained in $1 \mathrm{ml}$. of a solution consisting of $1 \mathrm{ml}$. of freshly prepared saturated sodium hydrosulphite per $6.5 \mathrm{ml}$. of $0.1 \%$ sodium carbonate. This solution had no immediate or delayed effect on the optical density of blood fully saturated with carbon monoxide, and it was concluded that no turbidity was formed. The oxygenated sample was obtained by rotation of the blood in a tonometer.

$(e)$ The light transmissions of the carbonate-saponin and carbonate-saponin-hydrosulphite solutions were almost identical, being $97-99 \%$ of that of saline.

$(f)$ In practice it was found to be unnecessary to flush out the cuvette with saline after every reading, and a series of samples could be run through in quick succession, each sample washing out the preceding one and taking its place. It was necessary, however, to flush out the cuvette with saline after the optical density of the reduced sample had been determined in order to remove all traces of hydrosulphite.

(g) The syringes used were made of nylon (" atlas," C. F. Thackray, Leeds) and the dilution 1:1 of the $1 \mathrm{ml}$. samples of blood using syringes of $3 \mathrm{ml}$. capacity was found to be sufficiently accurate to allow reproducible readings to be obtained with successive samples of the same blood. The plunger of these syringes remains unmoved on tilting; this facilitates manipulation.

(h) The addition of sodium hydrosulphite to blood containing Evans blue dye (T.1824) bleaches the dye and alters the optical density. Estimations of oxygen saturation and content can be made in the presence of dye if the optical densities of reduced and oxygenated blood samples are measured before the dye is administered. The optical density values of the subsequent test samples (containing dye) can then be corrected for the contribution to the optical density according to the difference between the density of an oxygenated sample (containing dye) and that of the preliminary oxygenated (undyed) sample.

(i) The equation used to calculate the percentage oxygen saturation makes the estimations independent of all pigments in the blood other than oxyhaemoglobin, provided that the optical density of these pigments is not affected by sodium hydrosulphite.

\section{Results}

The calibration curve from which oxygen saturation is deduced from optical density measurements was constructed from observations made on 100 blood samples ranging in haemoglobin content from 12.2 to $18.4 \mathrm{~g}$. $/ 100 \mathrm{ml}$. of blood. The samples (10 or $20 \mathrm{ml}$.) were taken from the antecubital vein. A series of 5 to 10 samples was taken from each of a number of subjects. Some samples were taken before and some during a period of circulatory arrest, the latter providing blood with low oxygen saturation. In some instances the forearm muscles were exercised during the period of arrest. The samples were taken into 10 or $20 \mathrm{ml}$. nylon syringes, the nozzle dead space of which had been filled with heparin $(1,000$ units $/ \mathrm{ml}$.) and on each of these samples parallel duplicate measurements were made of the oxygen saturation using the manometric Van Slyke apparatus, and of the optical density determined as described above. One fully saturated and one fully reduced value was obtained from pooled samples of all the specimens of any one series from a given subject. The oxygen saturation values ranged from $15 \%$ to $98 \%$. Fig. 2 shows the values obtained by the Van Slyke method plotted against the optical density measurements. The standard deviation of the individual values from the line drawn by inspection was $1.85 \%$ saturation, and this line (Fig. 2) was used in the subsequent transference of optical density ratio into percentage oxygen saturation. A reasonably good fit to the points was obtained with the equation:

$$
Y=56.0572 X+31.2329 X^{2}+8.7771 \text {, }
$$

where $Y$ is the Van Slyke value and $X$ the optical density. The standard deviation from the regression 
line was $1.96 \%$ saturation. This curve, however, deviated from the actual points in the $95-100 \%$ saturation range, and as the line drawn by inspection was quite adequate for practical use no attempt was made to obtain a curve of better fit, as for example by the use of a cubic equation.

The oxygen capacities of 57 further samples of freshly drawn venous blood were determined by the Van Slyke technique and plotted against the difference in optical densities between reduced and oxygenated samples (Fig. 3). Some of these samples were diluted with saline to lower their oxygen capacity. High oxygen capacity values were obtained by using less than $1 \mathrm{ml}$. of carbonate saponin solution to haemolyse the blood. The Van Slyke determinations ranged from 4.5 to $30 \mathrm{vol.} \%$. The linear regression of the points so determined is expressed by the equation

$$
\mathrm{Y}=29.83 \mathrm{X}-0.385 \text {, }
$$

where $\mathrm{Y}$ is the Van Slyke value and $\mathrm{X}$ the difference in optical density between reduced and oxygenated samples. The standard ceviation from the regression line was 0.48 vol. $\%$.

The values for percentage oxygen saturation and oxygen capacity determined with the photoelectric technique were used to calculate the oxygen content. The oxygen contents of 93 samples calculated in this way have been compared with the results of Van Slyke analyses on the same samples (Fig. 4), and the standard deviation of the difference between the two methods was found to be 0.39 vol. $\%$.

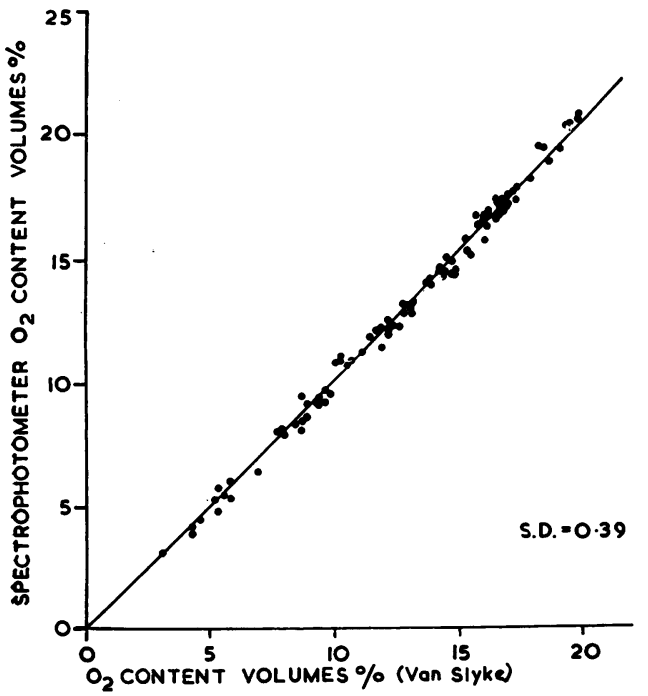

Frg. 4.-Comparison of 97 estimations of blood oxygen content by the photoelectric and Van Slyke methods. S.D. = standard deviation of the differences between the values obtained by the two methods. The line of identity is also shown.

\section{Discussion}

The type of cuvette described could easily be $\stackrel{\vec{F}}{\stackrel{\vec{S}}{+}}$ adapted to fit any other suitable instrument, the $\overline{0}$ chamber depth or the dilution of the sample being $\underline{\overline{ }}$. adjusted to permit readings on the most sensitive $\frac{\bar{\sigma}}{\vec{D}}$ part of the scale. For example, satisfactory results $\stackrel{\mathbb{\Omega}}{\propto}$ have been obtained by mounting a scaled-down version of the cuvette in an EEL colorimeter $\vec{O}$ (Evans Electro Selenium Ltd., Essex).

Since the percentage saturation is derived from $\overrightarrow{\vec{\omega}}$ a proportionality equation the calibration curve $\stackrel{S}{S}$ (Fig. 2) should apply to any instrument used. $\frac{\widehat{O}}{0}$ However, the fact that the curve departs from linearity, especially in the region $95-100 \%$ (Wood, i 1950), makes it advisable to check at least a few $\overrightarrow{-}$ points with any new cuvette and absorptiometer $\vec{G}$ combination to be used. It is always necessary 을 to obtain a calibration curve for capacity for each $\overrightarrow{7}$ instrument and cuvette.

The present technique does not involve the use of isobestic points for oxygenated and reduced $\overrightarrow{0}$ haemoglobin in the measurement of capacity, of readings being made at the same wavelength for 0 capacity and percentage saturation estimations. \& The use of an isobestic point to obtain capacity requires monochromatic light, the readings so obtained are not independent of other pigments, $\stackrel{\circ}{\stackrel{Q}{Q}}$ and a wavelength must be used which is different $\underset{\vec{A}}{\vec{A}}$ from that used for the percentage saturation $\frac{\overrightarrow{0}}{3}$ estimation.

The advantage of photoelectric over gasometric methods for the measurement of percentage oxygen saturation of blood lies in the rapidity with which the photoelectric determinations can be made. The rapidity of estimations is further increased in the present method since the cuvette is not removedo from the instrument and all readings are made at $₹$

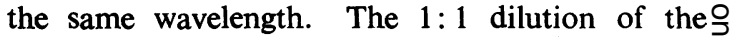
sample minimizes errors due to gaseous exchange $>$ between the blood and the diluting fluid. The accuracy of the results compares favourably with those obtained by other photometric methods (Wood and Cronin, 1953).

The method has been used in the measurement $\omega$ of the oxygen saturation of blood withdrawn during cardiac catheterization and of samples taken duringe such procedures as reactive hyperaemia (McNeill, 1956), indirect heating, and nerve block of the? forearm (Roddie, Shepherd, and Whelan, 1956, 궁 1957), as many as 150 samples being analysed $\stackrel{\vec{D}}{\circ}$ during the course of an experiment lasting two to? three hours. During such investigations the optical $\stackrel{\odot}{\perp}$ density of an oxygenated and a reduced sampleo are determined at the start of the procedure. The subsequent test samples are drawn directly into음 
nylon syringes and the optical densities determined immediately on withdrawal. This can be done in a few seconds for each sample and enables the course of the investigation to be adjusted in the light of the blood changes.

\section{Summary}

A spectrophotometric method is described for the estimation of blood oxygen saturation, content, and capacity.

The method involves the use of a glass cuvette which remains fixed in a standard absorptiometer, a 1:1 dilution of $1 \mathrm{ml}$. blood samples, and a wavelength setting of $660 \mathrm{~m} \mu$.

The main advantage of the method lies in the rapidity with which the measurements can be made.

Calibration curves for percentage oxygen saturation and for oxygen capacity have been construsted by comparison of the optical density values with monometric Van Slyke measurements.

In 100 blood samples ranging in $\mathrm{Hb}$ concentration from 12.2 to $18.4 \mathrm{~g} . / 100 \mathrm{ml}$. blood, with percentage saturations ranging from 15 to 98 , the standard deviation of the differences between values obtained by the two methods was $1.85 \%$ saturation. In 57 comparisons of capacity with Van Slyke determinations ranging from 4.5 to $30 \mathrm{vol} . \%$ the standard deviation was 0.48 vol. $\%$. In 93 comparisons of content with Van Slyke determinations ranging from 3 to 20 vol. $\%$ the standard deviation was 0.39 vol. $\%$.

We thank Professor A. D. M. Greenfield and Professor D. C. Harrison for advice and criticism in the preparation of the paper. We are grateful to Dr. E. A. Cheeseman and Mr. J. D. Merrett, of the Department of Social and Preventive Medicine, Queen's University, Belfast, for advice and assistance with some of the statistics. We thank Mr. L. M. Speet, Department of Physics, who made the glass cuvette.

\section{REFERENCES}

Drabkin, D. L. (1950). In Medical Physics, ed. O. Glasser, Vol. 2, p. 1039. Year Book Publishers, Chicago.

and Austin, J. H. (1935). J. biol. Chem., 112, 105.

Handforth C. P. (1952) Lancet 11252

Hickam, J. B., and Frayser, R. (1949). J. biol. Chem., 180, 457.

Horecker, B. L. (1943). Ibid., 148, 173.

Kramer, K. (1934). Z. Biol., 95, 126.

Elam, J. O., Saxton, G. A., and Elam, W. N. (1951). Amer. J. Physiol., 165, 229.

McNeill, T. A. (1956), J. Physiol. (Lond.), 134, 195.

Matthes, K., and Gross, F. (1939). Arch. exp. Path. Pharmak., 191, 523.

Molyneux, L., and Pask, E. A. (1955). Brit. Heart J., 17, 169.

Nahas, G. G. (1951). Science, 113, 723.

Nicolai, L. (1932). Pflügers Arch. ges. Physiol., 229, 372.

Roddie, I. C., Shepherd, J. T., and Whelan, R. F. (1956). J. Physiol. (Lond.), 134, 444.

(1957). Clin. Sci., 16, 67.

ade, O. L., Bishop, J M., Cumming, G., and Donald, K. W. (1953). Brit. med. J., $2,902$.

Wood, E. H. (1950). In Medical Physics, ed. O. Glasser, Vol. 2, p. 664. Year Book Publishers, Chicago.

and Cronin, L. (1953). Abstracts of Communications, 19th International Physiological Congress, Montreal, p. 898. 